\title{
IMMUNO-MEDIATED NECROTIZING MYOPATHY ASSOCIATED WITH POSITIVE ANTI-SRP ANTIBODY
}

Giulia Mazzuco Dallabrida ${ }^{1, \star}$, Guilherme Sanchez Corrêa², Ivânio Alves Pereira ${ }^{1}$

1.Universidade do Sul de Santa Catarina, Palhoça (SC), Brazil. 2.Universidade Federal de Santa Catarina, Florianópolis (SC), Brazil.

*Corresponding author: giuliadallabrida@gmail.com

\section{BACKGROUND}

Inflammatory myopathies are a heterogeneous group of diseases that include: polymyositis, dermatomyositis, inclusion body myositis and immune-mediated necrotizing myopathy (IMNM). Immune-mediated necrotizing myopathy is characterized by necrotic fibers with minimal lymphocytic infiltrate, high elevations of muscle enzymes and may be associated with anti-SRP and anti-HMGCR antibodies (related to statins).

\section{CASE REPORT}

E.Z.O., female, 51 years old, housewife and resident of Florianópolis. Six months earlier, she started with muscle weakness in the upper and lower limbs, with difficulty raising her arms. Associated with the condition, she reported dysphagia, alopecia, bilateral hand paresthesia and loss of $6 \mathrm{~kg}$ in three months. In physical examination, she had grade 4 strength in all four limbs. The exams showed $4309 \mathrm{U} / \mathrm{L}$ creatine phosphokinase (normal: 10-170), $948 \mathrm{U} / \mathrm{L}$ lactic dehydrogenase (reference <50), $41.5 \mathrm{U} / \mathrm{L}$ aldolase (<7.6), 164 U/L aspartate aminotransferase $(<32)$ and $189 \mathrm{U} / \mathrm{L}$ alanine aminotransferase $(<33)$. The antinuclear factor exhibited a dense thin cytoplasmic pattern, with negative anti-Ro, anti-La, anti-MI2, anti-jo1, anti-PL7, anti-PL12 and anti-HMGCR and positive anti-SRP. The search for malignancies with imaging, endoscopy and colonoscopy was negative. Electromyography showed a short-lived polyphasic pattern in proximal muscles. Magnetic resonance imaging showed signs of mild myopathy of the thigh and arm muscles, diffuse muscle hypotrophy and slight peripheral contrast impregnation. A biopsy of the quadriceps femoris muscle was performed, which resulted in the presence of necrotic fibers, a significant variation in the caliber of the muscle fibers due to capillary wall regeneration and thickening (Fig. 1). The immunohistochemical study revealed enhancement of capillaries and necrotic fibers, without significant lymphocytic infiltrate (Fig. 2). The patient started treatment with oral prednisone, with replacement by methotrexate and, in the face of therapeutic failure, association with azathioprine and rituximab. Currently, she is using intravenous immunoglobulin, with a stable clinical picture.

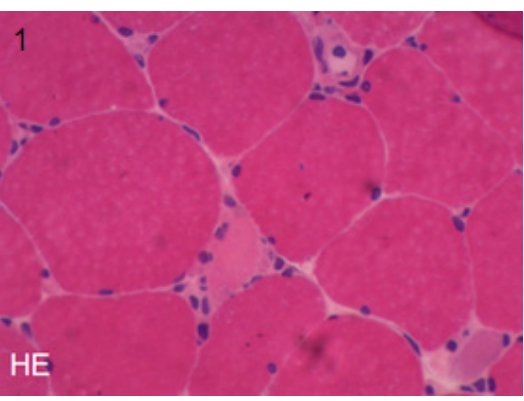

Figure 1. Biopsy of the quadriceps femoris muscle, hematoxylin and eosin staining: necrotic fibers surrounded by myophagocytes and a significant variation in the caliber of the muscle fibers.

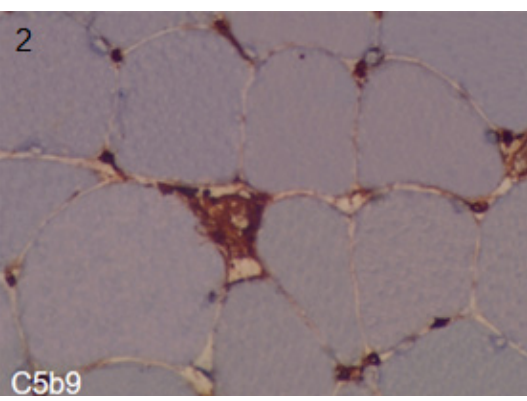

Figure 2. Staining of C5b-9: enhancement of capillaries and necrotic fibers.

\section{CONCLUSION}

Immune-mediated necrotizing myopathies are rare and only $10 \%$ have positive anti-SRP or anti-HMGCR. The prognosis is the worst of the myopathy subtypes, with persistence of weakness after intense immunosuppressive treatment.

\section{ACKNOWLEDEGMENTS}

Nathália de Assunção provided technical and writing assistance. 